\title{
Investigation of $\mathrm{A}^{+}$-States in $\mathrm{Si}$ and $\mathrm{Ge}$ by Phonon-Induced Conduction Under Uniaxial Stress
}

P. Gross and K. Lassmann

1. Physikalisches Institut, Universität Stuttgart, W-7000 Stuttgart 80, Fed. Rep. of Germany

From optical experiments (see for example [1,2]) it is known that acceptor bound excitons in $\mathrm{Si}\left(\mathrm{A}^{0} \mathrm{X}\right)$ have a ground state multiplet with a splitting in the meV-range. In some models the splitting was ascribed to the Coulomb interaction (jj-coupling) between the holes. In such a model the two $j=\frac{3}{2}$-holes couple to a total angular momentum of $J=0$ and $J=2$, respectively $\left(\Gamma_{1}\right.$ and $\Gamma_{3}+\Gamma_{5}$, respectively, in $T_{d} ; J=1$ is Pauli-forbidden). In a cubic field the $J=$ 2-state can be further split into $\Gamma_{3}$ and $\Gamma_{5}$. The contribution of the electron was neglected. If this model is correct, there should be a comparable splitting in the case of the corresponding $\mathrm{A}^{+}$-states. By the analysis of the stress dependence of phonon induced conductivity (PIC, $[3,4]$ ) with superconducting Al-tunneling junctions as phonon generators we have now found strong evidence for a split ground state in the cases $\mathrm{Si}: \mathrm{Ga}^{+}, \mathrm{Si}: \mathrm{Al}^{+}$and $\mathrm{Ge}: \mathrm{Zn}^{+}$.

The stress dependence of the $\mathrm{A}^{+}$-related PIC-thresholds should indicate whether there is a considerable hole-hole coupling or not: if the coupling is very small (independent holes) the stress splitting $\Delta^{\boldsymbol{A}}$ of the $\mathrm{A}^{+}$-ground state (two-hole state) is linear extrapolating back to zero splitting at zero stress. In this limit the two-hole state can be built up from independent one-hole $\left(\Gamma_{8^{-}}\right)$states. A $\Gamma_{\mathbf{B}^{-}}$-level splits under stress in two levels, which each can take up two holes. The sixfold degenerate $\Gamma_{8} \times \Gamma_{8}$-state then splits under stress into three levels: the lowest two-hole level corresponds to the case of two holes in the lower one-hole level, the fourfold degenerate middle two-hole level corresponds to the case of a hole in the lower and the upper one-hole level, and the highest two-hole level corresponds to the case of two holes in the upper one-hole level. A transition from the lowest to the highest two-hole state would mean a simultaneous excitation of two holes by one phonon and is not allowed for the one-particle phonon interaction.

On the other hand, if the coupling is not negligible, there is a zero stress splitting $\delta$ and the stress splitting is nonlinear: $\sqrt{\frac{\delta^{2}}{4}+\left(\Delta^{A}\right)^{2}}$. The splitting becomes again linear for stress splittings $\Delta^{\wedge}$ much larger than the hole-hole coupling $\delta$. In this limit, the holes are again decoupled. 


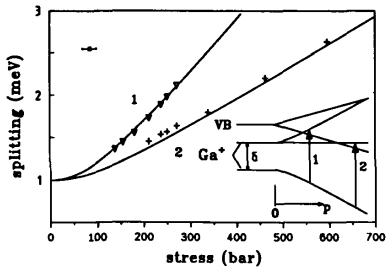

Fig.1: Shift of the signal maxima due to crossings with VB for $\mathrm{Si}: \mathrm{Ga}^{+}$. Also shown is the model for the $\mathrm{Ga}^{+}$-ground state.

In our PIC experiments we find as a common feature for the $\mathrm{A}^{+}$-states $\mathrm{B}^{+}$, $\mathrm{Al}^{+}, \mathrm{Ga}^{+}$in $\mathrm{Si}$ and $\mathrm{Zn}^{+}, \mathrm{Be}^{+}$in $\mathrm{Ge}$ a sharp line emerging at certain stresses at the low energy side of the threshold (see [4]). This line is interpreted as the crossing of one of the upper split $\mathrm{A}^{+}$-states with the valence band. The signal maximum in this situation corresponds to a phonon induced transition within the $\mathrm{A}^{+}$-state. If the upper $\mathrm{A}^{+}$-state is resonant with the valence band, the excited hole can escape to the band and a PIC-signal can be detected. The shift of the maximum of the line corresponds directly to the stress shift of the $\mathrm{A}^{+}$-splitting.

In the cases $\mathrm{Si}: \mathrm{B}^{+}$and $\mathrm{Ge}: \mathrm{Be}^{+}$, we find one crossing with the valence band, respectively. In both cases the splitting is linear extrapolating back to zero splitting at zero stress, that is, the hole-hole coupling can be neglected.

In the case Si:Ga+ (Fig. 1) we find two crossings with the valence band and a nonlinear shift of the corresponding signal maxima. This behaviour can be understood, if a split $\mathrm{Ga}^{+}$-ground state is assumed. In this case we can observe transitions from the lowest $\mathrm{A}^{+}$-state to both of the excited states as long as the holes are not decoupled. Hence, the signal due to the transition from the lowest level to the highest level should vanish at high stresses. This is indeed observed experimentally. We can fit the stress shift of the transitions with the above mentioned square root dependence. From the fit we get $\delta=1 \mathrm{meV}$. In a similar way, we get $\delta=1.2 \mathrm{meV}$ for $\mathrm{Si}: \mathrm{Al}^{+}$and $\delta=1.4 \mathrm{meV}$ for $\mathrm{Ge}: \mathrm{Zn}^{+}$. Financial support from the Deutsche Forschungsgemeinschaft is gratefully acknowledged.

[1] K.R. Elliott, G.C. Osbourn, D.L. Smith, T.C. McGill: Phys. Rev. B17, 1808 (1978)

[2] M.A. Vouk, E.C. Lightowlers: J. Lumines. 15, 357 (1977)

[3] W. Burger, K. Laßmann: Phys. Rev. Lett. 53, 2035 (1984)

[4] P. GroB, M. Gienger, K. Laßmann: Jap. J. Appl. Phys. 26 (1987) Supplement 26-3 (p.673) 